\title{
Third-Order Differential Subordination and Superordination Results for Meromorphically Multivalent Functions Associated with the Liu-Srivastava Operator
}

\author{
Huo Tang, ${ }^{1,2}$ H. M. Srivastava, ${ }^{3}$ Shu-Hai Li, ${ }^{1}$ and Li-Na Ma ${ }^{1}$ \\ ${ }^{1}$ School of Mathematics and Statistics, Chifeng University, Chifeng, Inner Mongolia 024000, China \\ ${ }^{2}$ School of Mathematical Sciences, Beijing Normal University, Beijing 100875, China \\ ${ }^{3}$ Department of Mathematics and Statistics, University of Victoria, Victoria, BC, Canada V8W 3R4
}

Correspondence should be addressed to Huo Tang; thth2009@tom.com and H. M. Srivastava; harimsri@math.uvic.ca

Received 14 April 2014; Revised 7 June 2014; Accepted 8 June 2014; Published 8 July 2014

Academic Editor: Om P. Ahuja

Copyright (C) 2014 Huo Tang et al. This is an open access article distributed under the Creative Commons Attribution License, which permits unrestricted use, distribution, and reproduction in any medium, provided the original work is properly cited.

\begin{abstract}
There are many articles in the literature dealing with the first-order and the second-order differential subordination and superordination problems for analytic functions in the unit disk, but only a few articles are dealing with the above problems in the third-order case (see, e.g., Antonino and Miller (2011) and Ponnusamy et al. (1992)). The concept of the third-order differential subordination in the unit disk was introduced by Antonino and Miller in (2011). Let $\Omega$ be a set in the complex plane $\mathbb{C}$. Also let $\mathfrak{p}$ be analytic in the unit disk $\mathbb{U}=\{z: z \in \mathbb{C}$ and $|z|<1\}$ and suppose that $\psi: \mathbb{C}^{4} \times \mathbb{U} \rightarrow \mathbb{C}$. In this paper, we investigate the problem of determining properties of functions $\mathfrak{p}(z)$ that satisfy the following third-order differential superordination: $\Omega \subset\left\{\psi\left(\mathfrak{p}(z), z \mathfrak{p}^{\prime}(z), z^{2} \mathfrak{p}^{\prime \prime}(z), z^{3} \mathfrak{p}^{\prime \prime \prime}(z) ; z\right): z \in \mathbb{U}\right\}$. As applications, we derive some third-order differential subordination and superordination results for meromorphically multivalent functions, which are defined by a family of convolution operators involving the Liu-Srivastava operator. The results are obtained by considering suitable classes of admissible functions.
\end{abstract}

\section{Introduction, Definitions, and Preliminaries}

Let $\mathscr{H}(\mathbb{U})$ be the class of functions which are analytic in the open unit disk:

$$
\mathbb{U}=\{z: z \in \mathbb{C},|z|<1\} .
$$

For $n \in \mathbb{N}:=\{1,2,3, \ldots\}$ and $a \in \mathbb{C}$, let

$$
\begin{aligned}
\mathscr{H}[a, n]=\{f: f \in \mathscr{H}(\mathbb{U}), \\
\left.\quad f(z)=a+a_{n} z^{n}+a_{n+1} z^{n+1}+\cdots\right\}
\end{aligned}
$$

and suppose that $\mathscr{H}=\mathscr{H}[1,1]$.

Let $f$ and $F$ be members of the analytic function class $\mathscr{H}(\mathbb{U})$. The function $f$ is said to be subordinate to $F$, or $F$ is superordinate to $f$, if there exists a Schwarz function $\mathfrak{w}(z)$, analytic in $\mathbb{U}$ with

$$
\mathfrak{w}(0)=0, \quad|\mathfrak{w}(z)|<1 \quad(z \in \mathbb{U}),
$$

such that

$$
f(z)=F(\mathfrak{w}(z)) .
$$

In such a case, we write

$$
f \prec F \quad \text { or } \quad f(z) \prec F(z) .
$$

Furthermore, if the function $F$ is univalent in $\mathbb{U}$, then we have the following equivalence (see, for details, [1]):

$$
\begin{aligned}
f(z) \prec F(z) & (z \in \mathbb{U}) \Longleftrightarrow f(0)=F(0), \\
& f(\mathbb{U}) \subset F(\mathbb{U}) .
\end{aligned}
$$

Let $\Sigma_{p}$ denote the class of functions of the form

$$
f(z)=\frac{1}{z^{p}}+\sum_{k=1-p}^{\infty} a_{k} z^{k} \quad(p \in \mathbb{N})
$$


which are analytic and multivalent in the punctured unit disk:

$$
\mathbb{U}^{*}=\{z \in \mathbb{C}: 0<|z|<1\}=\mathbb{U} \backslash\{0\} .
$$
by

For the function $f$ given by $(7)$ and the function $g$ given

$$
g(z)=\frac{1}{z^{p}}+\sum_{k=1-p}^{\infty} b_{k} z^{k} \quad\left(p \in \mathbb{N} ; z \in \mathbb{U}^{*}\right),
$$

the Hadamard product (or convolution) $f * g$ of the functions $f$ and $g$ is defined by

$$
(f * g)(z):=\frac{1}{z^{p}}+\sum_{k=1-p}^{\infty} a_{k} b_{k} z^{k}=:(g * f)(z) .
$$

For parameters $\alpha_{i} \in \mathbb{C}(i=1,2, \ldots, q)$ and $\beta_{j} \in$ $\mathbb{C} \backslash \mathbb{Z}_{0}^{-}\left(\mathbb{Z}_{0}^{-}=0,-1,-2, \ldots ; j=1,2, \ldots, s\right)$, the generalized hypergeometric function ${ }_{q} F_{s}\left(\alpha_{1}, \ldots, \alpha_{q} ; \beta_{1}, \ldots, \beta_{s} ; z\right)$ is defined by (see, for example, $[2,3]$ )

$$
\begin{array}{r}
{ }_{q} F_{s}\left(\alpha_{1}, \ldots, \alpha_{q} ; \beta_{1}, \ldots, \beta_{s} ; z\right)=\sum_{k=0}^{\infty} \frac{\left(\alpha_{1}\right)_{k} \cdots\left(\alpha_{q}\right)_{k}}{\left(\beta_{1}\right)_{k} \cdots\left(\beta_{s}\right)_{k}} \frac{z^{k}}{k !} \\
\left(q \leq s+1 ; q, s \in \mathbb{N}_{0}=\mathbb{N} \cup\{0\} ; z \in \mathbb{U}\right),
\end{array}
$$

where $(\nu)_{k}$ denotes the Pochhammer symbol defined, in terms of Gamma function, by

$$
\begin{aligned}
(\nu)_{k} & =\frac{\Gamma(\nu+k)}{\Gamma(\nu)} \\
& = \begin{cases}1 & (k=0 ; \nu \in \mathbb{C} \backslash\{0\}), \\
\nu(\nu+1) \cdots(\nu+k-1) & (k \in \mathbb{N} ; \nu \in \mathbb{C}) .\end{cases}
\end{aligned}
$$

Recently, Tang et al. [4] introduced a function $h_{p}^{\lambda, \mu}\left(\alpha_{1}, \ldots, \alpha_{q} ; \beta_{1}, \ldots, \beta_{s} ; z\right)$ defined by

$$
\begin{aligned}
& h_{p}^{\lambda, \mu}\left(\alpha_{1}, \ldots, \alpha_{q} ; \beta_{1}, \ldots, \beta_{s} ; z\right) \\
& =(1-\lambda+\mu) z_{q}^{-p} F_{s}\left(\alpha_{1}, \ldots, \alpha_{q} ; \beta_{1}, \ldots, \beta_{s} ; z\right) \\
& +(\lambda-\mu) z\left[z_{q}^{-p} F_{s}\left(\alpha_{1}, \ldots, \alpha_{q} ; \beta_{1}, \ldots, \beta_{s} ; z\right)\right]^{\prime} \\
& +\lambda \mu z^{2}\left[z_{q}^{-p} F_{s}\left(\alpha_{1}, \ldots, \alpha_{q} ; \beta_{1}, \ldots, \beta_{s} ; z\right)\right]^{\prime \prime} \\
& (p \in \mathbb{N} ; \lambda, \mu \geq 0 ; z \in \mathbb{U}) .
\end{aligned}
$$

In particular, when $\lambda=\mu=0$, we obtain

$$
h_{p}^{0,0}\left(\alpha_{1}, \ldots, \alpha_{q} ; \beta_{1}, \ldots, \beta_{s} ; z\right)=h_{p}\left(\alpha_{1}, \ldots, \alpha_{q} ; \beta_{1}, \ldots, \beta_{s} ; z\right) \text {, }
$$

which was introduced and studied by Liu and Srivastava [5].

Corresponding to the function $h_{p}^{\lambda, \mu}\left(\alpha_{1}, \ldots, \alpha_{q} ; \beta_{1}, \ldots, \beta_{s} ; z\right)$ given by (13), we consider a convolution operator

$$
H_{p}^{\lambda, \mu}\left(\alpha_{1}, \ldots, \alpha_{q} ; \beta_{1}, \ldots, \beta_{s}\right): \Sigma_{p} \longrightarrow \Sigma_{p}
$$

defined by the following Hadamard product (or convolution):

$$
\begin{aligned}
H_{p}^{\lambda, \mu} & \left(\alpha_{1}, \ldots, \alpha_{q} ; \beta_{1}, \ldots, \beta_{s}\right) f(z) \\
& =h_{p}^{\lambda, \mu}\left(\alpha_{1}, \ldots, \alpha_{q} ; \beta_{1}, \ldots, \beta_{s} ; z\right) * f(z) .
\end{aligned}
$$

For the sake of convenience, we write

$$
H_{p, q, s}^{\lambda, \mu}\left(\beta_{1}\right)=H_{p, q, s}^{\lambda, \mu}\left(\alpha_{1}\right)=H_{p}^{\lambda, \mu}\left(\alpha_{1}, \ldots, \alpha_{q} ; \beta_{1}, \ldots, \beta_{s}\right) .
$$

It is easily verified from definition (16) that

$$
\begin{aligned}
z\left(H_{p, q, s}^{\lambda, \mu}\left(\beta_{1}+1\right) f(z)\right)^{\prime}= & \beta_{1} H_{p, q, s}^{\lambda, \mu}\left(\beta_{1}\right) f(z) \\
& -\left(\beta_{1}+p\right) H_{p, q, s}^{\lambda, \mu}\left(\beta_{1}+1\right) f(z), \\
z\left(H_{p, q, s}^{\lambda, \mu}\left(\alpha_{1}\right) f(z)\right)^{\prime}= & \alpha_{1} H_{p, q, s}^{\lambda, \mu}\left(\alpha_{1}+1\right) f(z) \\
& -\left(\alpha_{1}+p\right) H_{p, q, s}^{\lambda, \mu}\left(\alpha_{1}\right) f(z) .
\end{aligned}
$$

We note that, for $\lambda=\mu=0$, the operator $H_{p, q, s}^{0,0}\left(\alpha_{1}\right)$ reduces to the Liu-Srivastava operator $H_{p, q, s}\left(\alpha_{1}\right)$ (see [5, 6]; see also [7]), while the Liu-Srivastava operator is the meromorphic analogous of the Dziok-Srivastava operator (see [8-10]; see also [11, 12]), which includes (as its special cases) the meromorphic analogous of the Carlson-Shaffer convolution operator $L_{p}(a, c)=H_{p, 2,1}^{0,0}(1, a ; c)$ (see $\left.[13,14]\right)$, the meromorphic analogous of the Ruscheweyh derivative operator $D^{n+1}=L_{p}(n+p, 1)$ (see [15]), and the operator

$$
J_{\delta, p}=\frac{\delta}{z^{\delta+p}} \int_{0}^{z} t^{\delta+p-1} f(t) d t=L_{p}(\delta, \delta+1) \quad(\delta>0)
$$

studied by Uralegaddi and Somanatha [16].

Let $\Omega$ be any set in $\mathbb{C}$. Also let $\mathfrak{p}$ be analytic in $\mathbb{U}$ and suppose that $\psi: \mathbb{C}^{4} \times \mathbb{U} \rightarrow \mathbb{C}$. Recently, Antonino and Miller [17] have extended the theory of second-order differential subordinations in $\mathbb{U}$ introduced by Miller and Mocanu [1] to the third-order case. They determined properties of functions $\mathfrak{p}(z)$ that satisfy the following third-order differential subordination:

$$
\left\{\psi\left(\mathfrak{p}(z), z \mathfrak{p}^{\prime}(z), z^{2} \mathfrak{p}^{\prime \prime}(z), z^{3} \mathfrak{p}^{\prime \prime \prime}(z) ; z\right): z \in \mathbb{U}\right\} \subset \Omega .
$$

We will now recall some definitions and a theorem due to Antonino and Miller [17], which are required in our next investigations.

Definition 1 (see [17], p. 440, Definition 1). Let $\psi: \mathbb{C}^{4} \times \mathbb{U} \rightarrow$ $\mathbb{C}$ and $h(z)$ be univalent in $\mathbb{U}$. If $\mathfrak{p}(z)$ is analytic in $\mathbb{U}$ and satisfies the following third-order differential subordination:

$$
\psi\left(\mathfrak{p}(z), z \mathfrak{p}^{\prime}(z), z^{2} \mathfrak{p}^{\prime \prime}(z), z^{3} \mathfrak{p}^{\prime \prime \prime}(z) ; z\right) \prec h(z),
$$


then $\mathfrak{p}(z)$ is called a solution of the differential subordination. A univalent function $\mathfrak{q}(z)$ is called a dominant of the solutions of the differential subordination or, more simply, a dominant if $\mathfrak{p}(z) \prec \mathfrak{q}(z)$ for all $\mathfrak{p}(z)$ satisfying (22). A dominant $\widetilde{\mathfrak{q}}(z)$ that satisfies $\widetilde{\mathfrak{q}}(z) \prec \mathfrak{q}(z)$ for all dominants $\mathfrak{q}(z)$ of $(22)$ is said to be the best dominant.

Definition 2 (see [17], p. 441, Definition 2). Let Q denote the set of functions $\mathfrak{q}$ that are analytic and univalent on the set $\overline{\mathbb{U}} \backslash E(\mathfrak{q})$, where

$$
E(\mathfrak{q})=\left\{\xi: \xi \in \partial \mathbb{U} \text { and } \lim _{z \rightarrow \xi} \mathfrak{q}(z)=\infty\right\},
$$

is such that

$$
\min \left|\mathfrak{q}^{\prime}(\xi)\right|=\rho>0
$$

for $\xi \in \partial \mathbb{U} \backslash E(\mathfrak{q})$. Further, let the subclass of $\mathbb{Q}$ for which $\mathfrak{q}(0)=a$ be denoted by $Q(a)$ and

$$
Q(1)=Q_{1} \text {. }
$$

Definition 3 (see [17], p. 449, Definition 3). Let $\Omega$ be a set in $\mathbb{C}, \mathfrak{q} \in \mathbb{Q}$, and $n \in \mathbb{N} \backslash\{1\}$. The class of admissible functions $\Psi_{n}[\Omega, \mathfrak{q}]$ consists of those functions $\psi: \mathbb{C}^{4} \times \mathbb{U} \rightarrow \mathbb{C}$ that satisfy the following admissibility condition:

$$
\psi(r, s, t, u ; z) \notin \Omega
$$

whenever

$$
\begin{gathered}
r=\mathfrak{q}(\xi), \quad s=k \xi \mathfrak{q}^{\prime}(\xi), \\
\mathfrak{R}\left(\frac{t}{s}+1\right) \geqq k \Re\left(\frac{\xi \mathfrak{q}^{\prime \prime}(\xi)}{\mathfrak{q}^{\prime}(\xi)}+1\right), \\
\Re\left(\frac{u}{s}\right) \geqq k^{2} \mathfrak{R}\left(\frac{\xi^{2} \mathfrak{q}^{\prime \prime \prime}(\xi)}{\mathfrak{q}^{\prime}(\xi)}\right),
\end{gathered}
$$

where $z \in \mathbb{U}, \xi \in \partial \mathbb{U} \backslash E(\mathfrak{q})$, and $k \geqq n$.

Theorem 4 (see [17], p. 449, Theorem 1). Let $\mathfrak{p} \in \mathscr{H}[a, n]$ with $n \in \mathbb{N} \backslash\{1\}$. Also let $\mathfrak{q} \in \mathbb{Q}(a)$ and satisfy the following conditions:

$$
\Re\left(\frac{\xi \mathfrak{q}^{\prime \prime}(\xi)}{\mathfrak{q}^{\prime}(\xi)}\right) \geqq 0, \quad\left|\frac{z \mathfrak{p}^{\prime}(z)}{\mathfrak{q}^{\prime}(\xi)}\right| \leqq k,
$$

where $z \in \mathbb{U}, \xi \in \partial \mathbb{U} \backslash E(\mathfrak{q})$, and $k \geqq n$. If $\Omega$ is a set in $\mathbb{C}$, $\psi \in \Psi_{n}[\Omega, \mathfrak{q}]$ and

$$
\psi\left(\mathfrak{p}(z), z \mathfrak{p}^{\prime}(z), z^{2} \mathfrak{p}^{\prime \prime}(z), z^{3} \mathfrak{p}^{\prime \prime \prime}(z) ; z\right) \in \Omega,
$$

then

$$
\mathfrak{p}(z) \prec \mathfrak{q}(z) .
$$

In this paper, following the theory of second-order differential superordinations in the unit disk introduced by Miller and Mocanu [18], we consider the dual problem of determining properties of functions $\mathfrak{p}(z)$ that satisfy the following third-order differential superordination:

$$
\Omega \subset\left\{\psi\left(\mathfrak{p}(z), z \mathfrak{p}^{\prime}(z), z^{2} \mathfrak{p}^{\prime \prime}(z), z^{3} \mathfrak{p}^{\prime \prime \prime}(z) ; z\right): z \in \mathbb{U}\right\} .
$$

In other words, we determine the conditions on $\Omega, \Delta$, and $\psi$ for which the following implication holds true:

$$
\begin{aligned}
\Omega & \subset\left\{\psi\left(\mathfrak{p}(z), z \mathfrak{p}^{\prime}(z), z^{2} \mathfrak{p}^{\prime \prime}(z), z^{3} \mathfrak{p}^{\prime \prime \prime}(z) ; z\right): z \in \mathbb{U}\right\} \\
& \Longrightarrow \Delta \subset \mathfrak{p}(\mathbb{U})
\end{aligned}
$$

where $\Delta$ is any set in $\mathbb{C}$.

If either $\Omega$ or $\Delta$ is a simply connected domain, then (32) can be rephrased in terms of superordination. If $\mathfrak{p}(z)$ is univalent in $\mathbb{U}$, and if $\Delta$ is a simply connected domain with $\Delta \neq \mathbb{C}$, then there is a conformal mapping $\mathfrak{q}$ of $\mathbb{U}$ onto $\Delta$ such that $\mathfrak{q}(0)=\mathfrak{p}(0)$. In this case, (32) can be rewritten as follows:

$$
\begin{aligned}
\Omega & \subset\left\{\psi\left(\mathfrak{p}(z), z \mathfrak{p}^{\prime}(z), z^{2} \mathfrak{p}^{\prime \prime}(z), z^{3} \mathfrak{p}^{\prime \prime \prime}(z) ; z\right): z \in \mathbb{U}\right\} \\
& \Longrightarrow \mathfrak{q}(z) \prec \mathfrak{p}(z) .
\end{aligned}
$$

If $\Omega$ is also a simply connected domain with $\Omega \neq \mathbb{C}$, then there is a conformal mapping $h$ of $\mathbb{U}$ onto $\Omega$ such that $h(0)=$ $\psi(\mathfrak{p}(0), 0,0,0 ; 0)$. In addition, if the function

$$
\psi\left(\mathfrak{p}(z), z \mathfrak{p}^{\prime}(z), z^{2} \mathfrak{p}^{\prime \prime}(z), z^{3} \mathfrak{p}^{\prime \prime \prime}(z) ; z\right)
$$

is univalent in $\mathbb{U}$, then (33) can be rewritten as

$$
\begin{aligned}
h(z) & \prec \psi\left(\mathfrak{p}(z), z \mathfrak{p}^{\prime}(z), z^{2} \mathfrak{p}^{\prime \prime}(z), z^{3} \mathfrak{p}^{\prime \prime \prime}(z) ; z\right) \\
& \Longrightarrow \mathfrak{q}(z) \prec \mathfrak{p}(z) .
\end{aligned}
$$

There are three key ingredients in the implication relationship (33): the differential operator $\psi$, the set $\Omega$, and the "dominating" function $\mathfrak{q}$. If two of these entities were given, one would hope to find conditions on the third entity so that (33) would be satisfied. In this paper, we start with a given set $\Omega$ and a given function $\mathfrak{q}$, and we then determine a set of "admissible" operators $\psi$ so that (33) holds true.

We first introduce the following definition.

Definition 5. Let $\psi: \mathbb{C}^{4} \times \mathbb{U} \rightarrow \mathbb{C}$ and the function $h(z)$ be analytic in $\mathbb{U}$. If the functions $\mathfrak{p}(z)$ and

$$
\psi\left(\mathfrak{p}(z), z \mathfrak{p}^{\prime}(z), z^{2} \mathfrak{p}^{\prime \prime}(z), z^{3} \mathfrak{p}^{\prime \prime \prime}(z) ; z\right)
$$

are univalent in $\mathbb{U}$ and satisfy the following third-order differential superordination:

$$
h(z) \prec \psi\left(\mathfrak{p}(z), z \mathfrak{p}^{\prime}(z), z^{2} \mathfrak{p}^{\prime \prime}(z), z^{3} \mathfrak{p}^{\prime \prime \prime}(z) ; z\right),
$$

then $\mathfrak{p}(z)$ is called a solution of the differential superordination. An analytic function $\mathfrak{q}(z)$ is called a subordinant of 
the solutions of the differential superordination or more simply a subordinant if $\mathfrak{q}(z) \prec \mathfrak{p}(z)$ for $\mathfrak{p}(z)$ satisfying (37). A univalent subordinant $\widetilde{\mathfrak{q}}(z)$ that satisfies the condition

$$
\mathfrak{q}(z) \prec \widetilde{\mathfrak{q}}(z)
$$

for all subordinants $\mathfrak{q}(z)$ of (37) is said to be the best subordinant. We note that the best subordinant is unique up to a rotation of $\mathbb{U}$.

For $\Omega$ a set in $\mathbb{C}$, with $\psi$ and $\mathfrak{p}$ as given in Definition 5 , we suppose that (37) is replaced by

$$
\Omega \subset\left\{\psi\left(\mathfrak{p}(z), z \mathfrak{p}^{\prime}(z), z^{2} \mathfrak{p}^{\prime \prime}(z), z^{3} \mathfrak{p}^{\prime \prime \prime}(z) ; z\right): z \in \mathbb{U}\right\} .
$$

Although this more general situation is a "differential containment," yet we also refer to it as a differential superordination, and the definitions of solution, subordinant, and best subordinant as given above can be extended to this more general case.

We will use the following lemma [[17], p. 445, Lemma D] from the theory of third-order differential subordinations in $\mathbb{U}$ to determine subordinants of the third-order differential superordinations.

Lemma 6 (see [17]). Let $\mathfrak{p} \in \mathbb{Q}(a)$, and let $\mathfrak{q}(z)=a+a_{n} z^{n}+$ $\cdots$ be analytic in $\mathbb{U}$ with $\mathfrak{q}(z) \neq a$ and $n \in \mathbb{N} \backslash\{1\}$. If $\mathfrak{q}$ is not subordinate to $\mathfrak{p}$, then there exists points $z_{0}=r_{0} e^{i \theta_{0}} \in \mathbb{U}$ and $\xi_{0} \in \partial \mathbb{U} \backslash E(\mathfrak{p})$, and an $m \geqq n$ for which $\mathfrak{q}\left(\mathbb{U}_{r_{0}}\right) \subset \mathfrak{p}(\mathbb{U})$,

(i) $\mathfrak{q}\left(z_{0}\right)=\mathfrak{p}\left(\xi_{0}\right)$,

(ii) $\mathfrak{R}\left(\xi_{0} \mathfrak{p}^{\prime \prime}\left(\xi_{0}\right) / \mathfrak{p}^{\prime}\left(\xi_{0}\right)\right) \geqq 0$ and $\left|z \mathfrak{q}^{\prime}(z) / \mathfrak{p}^{\prime}\left(\xi_{0}\right)\right| \leqq m$,

(iii) $z_{0} \mathfrak{q}^{\prime}\left(z_{0}\right)=m \xi_{0} \mathfrak{p}^{\prime}\left(\xi_{0}\right)$,

(iv) $\mathfrak{R}\left(1+z_{0} \mathfrak{q}^{\prime \prime}\left(z_{0}\right) / \mathfrak{q}^{\prime}\left(z_{0}\right)\right) \geqq m \mathfrak{R}\left(1+\xi_{0} \mathfrak{p}^{\prime \prime}\left(\xi_{0}\right) / \mathfrak{p}^{\prime}\left(\xi_{0}\right)\right)$,

(v) $\mathfrak{R}\left(z_{0}^{2} \mathfrak{q}^{\prime \prime \prime}\left(z_{0}\right) / \mathfrak{q}^{\prime}\left(z_{0}\right)\right) \geqq m^{2} \mathfrak{R}\left(\xi_{0}^{2} \mathfrak{p}^{\prime \prime \prime}\left(\xi_{0}\right) / \mathfrak{p}^{\prime}\left(\xi_{0}\right)\right)$.

\section{Admissible Functions and a Fundamental Result}

We next define the class of admissible functions referred to in the preceding section.

Definition 7. Let $\Omega$ be a set in $\mathbb{C}, \mathfrak{q} \in \mathscr{H}[a, n]$ and $\mathfrak{q}^{\prime}(z) \neq 0$. The class of admissible functions $\Psi_{n}^{\prime}[\Omega, \mathfrak{q}]$ consists of those functions $\psi: \mathbb{C}^{4} \times \overline{\mathbb{U}} \rightarrow \mathbb{C}$ that satisfy the following admissibility condition:

$$
\psi(r, s, t, u ; \xi) \in \Omega
$$

whenever

$$
\begin{gathered}
r=\mathfrak{q}(z), \quad s=\frac{z \mathfrak{q}^{\prime}(z)}{m}, \\
\Re\left(\frac{t}{s}+1\right) \leqq \frac{1}{m} \mathfrak{R}\left(\frac{z \mathfrak{q}^{\prime \prime}(z)}{\mathfrak{q}^{\prime}(z)}+1\right), \\
\Re\left(\frac{u}{s}\right) \leqq \frac{1}{m^{2}} \mathfrak{R}\left(\frac{z^{2} \mathfrak{q}^{\prime \prime \prime}(z)}{\mathfrak{q}^{\prime}(z)}\right),
\end{gathered}
$$

If $\psi: \mathbb{C}^{2} \times \overline{\mathbb{U}} \rightarrow \mathbb{C}$ and $\mathfrak{q} \in \mathscr{H}[a, n]$, then the admissibility condition (41) reduces to the following form:

$$
\psi\left(\mathfrak{q}(z), \frac{z \mathfrak{q}^{\prime}(z)}{m} ; \xi\right) \in \Omega \quad(z \in \mathbb{U} ; \xi \in \partial \mathbb{U} ; m \geqq n \geqq 2) .
$$

If $\psi: \mathbb{C}^{3} \times \overline{\mathbb{U}} \rightarrow \mathbb{C}$ and $\mathfrak{q} \in \mathscr{H}[a, n]$ with $\mathfrak{q}^{\prime}(z) \neq 0$, then the admissibility condition (41) reduces to the following form:

$$
\psi(r, s, t ; \xi) \in \Omega
$$

whenever $r=\mathfrak{q}(z), s=z \mathfrak{q}^{\prime}(z) / m$, and

$$
\begin{array}{r}
\mathfrak{R}\left(\frac{t}{s}+1\right) \leqq \frac{1}{m} \mathfrak{R}\left(\frac{z \mathfrak{q}^{\prime \prime}(z)}{\mathfrak{q}^{\prime}(z)}+1\right) \\
(z \in \mathbb{U} ; \xi \in \partial \mathbb{U} ; m \geqq n \geqq 2) .
\end{array}
$$

The next theorem is a foundation result in the theory of the third-order differential superordinations in $\mathbb{U}$.

Theorem 8. Let $\mathfrak{q} \in \mathscr{H}[a, n]$ and $\psi \in \Psi_{n}^{\prime}[\Omega, \mathfrak{q}]$. If

$$
\psi\left(\mathfrak{p}(z), z \mathfrak{p}^{\prime}(z), z^{2} \mathfrak{p}^{\prime \prime}(z), z^{3} \mathfrak{p}^{\prime \prime \prime}(z) ; z\right)
$$

is univalent in $\mathbb{U}$ and $\mathfrak{p} \in \mathbb{Q}(a)$ satisfy the following conditions:

$$
\begin{array}{r}
\mathfrak{R}\left(\frac{z \mathfrak{q}^{\prime \prime}(z)}{\mathfrak{q}^{\prime}(z)}\right) \geqq 0, \quad\left|\frac{z \mathfrak{p}^{\prime}(z)}{\mathfrak{q}^{\prime}(z)}\right| \leqq m \\
(z \in \mathbb{U} ; \quad \xi \in \partial \mathbb{U} ; m \geqq n \geqq 2),
\end{array}
$$

then

$$
\Omega \subset\left\{\psi\left(\mathfrak{p}(z), z \mathfrak{p}^{\prime}(z), z^{2} \mathfrak{p}^{\prime \prime}(z), z^{3} \mathfrak{p}^{\prime \prime \prime}(z) ; z\right): z \in \mathbb{U}\right\}
$$

implies that

$$
\mathfrak{q}(z) \prec \mathfrak{p}(z)
$$

Proof. Suppose that

$$
\mathfrak{q}(z) \nprec \mathfrak{p}(z) .
$$

Then, by the above lemma, there exists points $z_{0}=r_{0} e^{i \theta_{0}} \in \mathbb{U}$ and $\xi_{0} \in \partial \mathbb{U} \backslash E(\mathfrak{p})$, and an $m \geqq n \geqq 2$ that satisfy conditions (i) $-(v)$ of the above lemma. Using these conditions with $r=\mathfrak{p}\left(\xi_{0}\right), s=\xi_{0} \mathfrak{p}^{\prime}\left(\xi_{0}\right), t=\xi_{0}^{2} \mathfrak{p}^{\prime \prime}\left(\xi_{0}\right), u=\xi_{0}^{3} \mathfrak{p}^{\prime \prime \prime}\left(\xi_{0}\right)$, and $\xi=\xi_{0}$ in Definition 7 , we obtain

$$
\psi\left(\mathfrak{p}\left(\xi_{0}\right), \xi_{0} \mathfrak{p}^{\prime}\left(\xi_{0}\right), \xi_{0}^{2} \mathfrak{p}^{\prime \prime}\left(\xi_{0}\right), \xi_{0}^{3} \mathfrak{p}^{\prime \prime \prime}\left(\xi_{0}\right) ; \xi_{0}\right) \in \Omega
$$

which contradicts (48), so we have

$$
\mathfrak{q}(z) \prec \mathfrak{p}(z) .
$$

where $z \in \mathbb{U}, \xi \in \partial \mathbb{U}$, and $m \geqq n \geqq 2$. 
In the special case when $\Omega \neq \mathbb{C}$ is a simply connected domain and $h$ is a conformal mapping of $\mathbb{U}$ onto $\Omega$, we denote this class $\Psi_{n}^{\prime}[h(\mathbb{U}), \mathfrak{q}]$ by $\Psi_{n}^{\prime}[h, \mathfrak{q}]$. The following result is an immediate consequence of Theorem 8 .

Theorem 9. Let $\mathfrak{q} \in \mathscr{H}[a, n]$. Also let the function $h$ be analytic in $\mathbb{U}$ and suppose that $\psi \in \Psi_{n}^{\prime}[h, \mathfrak{q}]$. If $\mathfrak{p} \in \mathbb{Q}(a)$ satisfies condition (47) and

$$
\psi\left(\mathfrak{p}(z), z \mathfrak{p}^{\prime}(z), z^{2} \mathfrak{p}^{\prime \prime}(z), z^{3} \mathfrak{p}^{\prime \prime \prime}(z) ; z\right)
$$

is univalent in $\mathbb{U}$, then

$$
h(z) \prec \psi\left(\mathfrak{p}(z), z \mathfrak{p}^{\prime}(z), z^{2} \mathfrak{p}^{\prime \prime}(z), z^{3} \mathfrak{p}^{\prime \prime \prime}(z) ; z\right)
$$

implies that

$$
\mathfrak{q}(z) \prec \mathfrak{p}(z)
$$

Theorems 8 and 9 can only be used to obtain subordinants of the third-order differential superordination of the forms (48) or (54).

Theorem 10. Let the function $h$ be analytic in $\mathbb{U}$ and let $\psi$ : $\mathbb{C}^{4} \times \overline{\mathbb{U}} \rightarrow \mathbb{C}$. Suppose that the differential equation

$$
\psi\left(\mathfrak{q}(z), z \mathfrak{q}^{\prime}(z), z^{2} \mathfrak{q}^{\prime \prime}(z), z^{3} \mathfrak{q}^{\prime \prime \prime}(z) ; z\right)=h(z)
$$

has a solution $\mathfrak{q} \in \mathbb{Q}(a)$. If $\psi \in \Psi_{n}^{\prime}[h, \mathfrak{q}], \mathfrak{p} \in \mathbb{Q}(a)$, and

$$
\psi\left(\mathfrak{p}(z), z \mathfrak{p}^{\prime}(z), z^{2} \mathfrak{p}^{\prime \prime}(z), z^{3} \mathfrak{p}^{\prime \prime \prime}(z) ; z\right)
$$

is univalent in $\mathbb{U}$, then (54) implies that

$$
\mathfrak{q}(z) \prec \mathfrak{p}(z)
$$

and $\mathfrak{q}(z)$ is the best subordinant.

Proof. Since $\psi \in \Psi_{n}^{\prime}[h, \mathfrak{q}]$, by applying Theorem 9, we deduce that $\mathfrak{q}$ is a subordinant of (54). Since $\mathfrak{q}$ satisfies (56), it is also a solution of the differential superordination (54). Therefore, all subordinants of (54) will be subordinate to $\mathfrak{q}$. It follows that $\mathfrak{q}(z)$ will be the best subordinant of (54).

In the next two sections, by making use of the thirdorder differential subordination results of Antonino and Miller [17] in the unit disk $\mathbb{U}$ and the third-order differential superordination results in $\mathbb{U}$ obtained in Section 2 (see, for details, Theorems 8, 9, and 10), we determine certain appropriate classes of admissible functions and investigate some third-order differential subordination and differential superordination properties of meromorphically multivalent functions associated with the operator $H_{p, q, s}^{\lambda, \mu}\left(\beta_{1}\right)$ defined by (16). It should be remarked in passing that, in recent years, several authors obtained many interesting results involving various linear and nonlinear convolution operators associated with (second-order) differential subordination and superordination, and the interested reader may refer to several earlier works including (for example) [19] to [20-23].

\section{Third-Order Differential Subordination of the Operator $H_{p, q, s}^{\lambda, \mu}\left(\beta_{1}\right)$}

We first define the following class of admissible functions, which are required in proving the differential subordination theorem involving the operator $H_{p, q, s}^{\lambda, \mu}\left(\beta_{1}\right)$ defined by (16).

Definition 11. Let $\Omega$ be a set in $\mathbb{C}$ and $\mathfrak{q} \in \mathbb{Q}_{1} \cap \mathscr{H}$. The class of admissible functions $\Phi_{H}[\Omega, \mathfrak{q}]$ consists of those functions $\phi: \mathbb{C}^{4} \times \mathbb{U} \rightarrow \mathbb{C}$ that satisfy the following admissibility condition:

$$
\phi(a, b, c, d ; z) \notin \Omega
$$

whenever

$$
\begin{gathered}
a=\mathfrak{q}(\xi), \quad b=\frac{k \xi \mathfrak{q}^{\prime}(\xi)+\beta_{1} \mathfrak{q}(\xi)}{\beta_{1}}, \\
\mathfrak{R}\left(\frac{\left(\beta_{1}+1\right)(c-a)}{b-a}-\left(2 \beta_{1}+1\right)\right) \geqq k \mathfrak{R}\left(\frac{\xi \mathfrak{q}^{\prime \prime}(\xi)}{\mathfrak{q}^{\prime}(\xi)}+1\right), \\
\mathfrak{R}\left(\frac{\left(\beta_{1}+1\right)\left(\beta_{1}+2\right)(d-3 c+3 b-a)}{b-a}\right) \\
\geqq k^{2} \mathfrak{R}\left\{\frac{\xi^{2} \mathfrak{q}^{\prime \prime \prime}(\xi)}{\mathfrak{q}^{\prime}(\xi)}\right\}
\end{gathered}
$$

where $z \in \mathbb{U}, \beta_{1} \in \mathbb{C} \backslash\{0,-1,-2, \ldots\}, \xi \in \partial \mathbb{U} \backslash E(\mathfrak{q})$, and $k \in \mathbb{N} \backslash\{1\}$.

Theorem 12. Let $\phi \in \Phi_{H}[\Omega, \mathfrak{q}]$. If the functions $f \in \Sigma_{p}$ and $\mathfrak{q} \in Q_{1}$ satisfy the following conditions:

$$
\begin{gathered}
\Re\left(\frac{\xi \mathfrak{q}^{\prime \prime}(\xi)}{\mathfrak{q}^{\prime}(\xi)}\right) \geqq 0, \quad\left|\frac{z^{p} H_{p, q, s}^{\lambda, \mu}\left(\beta_{1}\right) f(z)}{\mathfrak{q}^{\prime}(\xi)}\right| \leqq k, \\
\left\{\phi \left(z^{p} H_{p, q, s}^{\lambda, \mu}\left(\beta_{1}+1\right) f(z), z^{p} H_{p, q, s}^{\lambda, \mu}\left(\beta_{1}\right) f(z),\right.\right. \\
z^{p} H_{p, q, s}^{\lambda, \mu}\left(\beta_{1}-1\right) f(z), \\
\left.\left.z^{p} H_{p, q, s}^{\lambda, \mu}\left(\beta_{1}-2\right) f(z) ; z\right): z \in \mathbb{U}\right\} \subset \Omega,
\end{gathered}
$$

then

$$
z^{p} H_{p, q, s}^{\lambda, \mu}\left(\beta_{1}+1\right) f(z) \prec \mathfrak{q}(z)
$$

Proof. Define the analytic function $\mathfrak{p}(z)$ in $\mathbb{U}$ by

$$
\mathfrak{p}(z)=z^{p} H_{p, q, s}^{\lambda, \mu}\left(\beta_{1}+1\right) f(z)
$$

Then, differentiating (64) with respect to $z$ and using (18), we have

$$
z^{p} H_{p, q, s}^{\lambda, \mu}\left(\beta_{1}\right) f(z)=\frac{z \mathfrak{p}^{\prime}(z)+\beta_{1} \mathfrak{p}(z)}{\beta_{1}} .
$$


Further computations show that

$$
\begin{aligned}
& z^{p} H_{p, q, s}^{\lambda, \mu}\left(\beta_{1}-1\right) f(z) \\
& =\frac{z^{2} \mathfrak{p}^{\prime \prime}(z)+2\left(\beta_{1}+1\right) z \mathfrak{p}^{\prime}(z)+\beta_{1}\left(\beta_{1}+1\right) \mathfrak{p}(z)}{\beta_{1}\left(\beta_{1}+1\right)} \\
& z^{p} H_{p, q, s}^{\lambda, \mu}\left(\beta_{1}-2\right) f(z) \\
& =\left(z^{3} \mathfrak{p}^{\prime \prime \prime}(z)+3\left(\beta_{1}+2\right) z^{2} \mathfrak{p}^{\prime \prime}(z)\right. \\
& \quad+3\left(\beta_{1}+1\right)\left(\beta_{1}+2\right) z \mathfrak{p}^{\prime}(z) \\
& \left.\quad+\beta_{1}\left(\beta_{1}+1\right)\left(\beta_{1}+2\right) \mathfrak{p}(z)\right) \\
& \quad \times\left(\beta_{1}\left(\beta_{1}+1\right)\left(\beta_{1}+2\right)\right)^{-1}
\end{aligned}
$$

We now define the transformation from $\mathbb{C}^{4}$ to $\mathbb{C}$ by

$$
\begin{gathered}
a(r, s, t, u)=r, \quad b(r, s, t, u)=\frac{s+\beta_{1} r}{\beta_{1}}, \\
c(r, s, t, u)=\frac{t+2\left(\beta_{1}+1\right) s+\beta_{1}\left(\beta_{1}+1\right) r}{\beta_{1}\left(\beta_{1}+1\right)}, \\
d(r, s, t, u) \\
=\left(u+3\left(\beta_{1}+2\right) t+3\left(\beta_{1}+1\right)\left(\beta_{1}+2\right) s\right. \\
\left.\quad+\beta_{1}\left(\beta_{1}+1\right)\left(\beta_{1}+2\right) r\right) \\
\begin{array}{c}
\text { Let } \quad\left(\beta_{1}\left(\beta_{1}+1\right)\left(\beta_{1}+2\right)\right)^{-1} . \\
=\phi(a, b, c, d ; z) \\
=\phi\left(r, \frac{s+\beta_{1} r}{\beta_{1}}, \frac{t+2\left(\beta_{1}+1\right) s+\beta_{1}\left(\beta_{1}+1\right) r}{\beta_{1}\left(\beta_{1}+1\right)}\right. \\
\left.\frac{u+3\left(\beta_{1}+2\right) t+3\left(\beta_{1}+1\right)\left(\beta_{1}+2\right) s+\beta_{1}\left(\beta_{1}+1\right)\left(\beta_{1}+2\right) r}{\beta_{1}\left(\beta_{1}+1\right)\left(\beta_{1}+2\right)} ; z\right) .
\end{array}
\end{gathered}
$$

The proof will make use of Theorem 4. Using (64) to (67), we find from (70) that

$$
\begin{gathered}
\psi\left(\mathfrak{p}(z), z \mathfrak{p}^{\prime}(z), z^{2} \mathfrak{p}^{\prime \prime}(z), z^{3} \mathfrak{p}^{\prime \prime \prime}(z) ; z\right) \\
=\phi\left(z^{p} H_{p, q, s}^{\lambda, \mu}\left(\beta_{1}+1\right) f(z)\right. \\
z^{p} H_{p, q, s}^{\lambda, \mu}\left(\beta_{1}\right) f(z), z^{p} H_{p, q, s}^{\lambda, \mu}\left(\beta_{1}-1\right) f(z), \\
\left.z^{p} H_{p, q, s}^{\lambda, \mu}\left(\beta_{1}-2\right) f(z) ; z\right) .
\end{gathered}
$$

Hence, clearly, (62) becomes

$$
\psi\left(\mathfrak{p}(z), z \mathfrak{p}^{\prime}(z), z^{2} \mathfrak{p}^{\prime \prime}(z), z^{3} \mathfrak{p}^{\prime \prime \prime}(z) ; z\right) \in \Omega
$$

We note that

$$
\begin{aligned}
& \frac{t}{s}+1=\frac{\left(\beta_{1}+1\right)(c-a)}{b-a}-\left(2 \beta_{1}+1\right), \\
& \frac{u}{s}=\frac{\left(\beta_{1}+1\right)\left(\beta_{1}+2\right)(d-3 c+3 b-a)}{b-a} .
\end{aligned}
$$

Thus, the admissibility condition for $\phi \in \Phi_{H}[\Omega, \mathfrak{q}]$ in Definition 11 is equivalent to the admissibility condition for $\psi \in \Psi_{2}[\Omega, \mathfrak{q}]$ as given in Definition 3 with $n=2$. Therefore, by using (61) and Theorem 4 , we have

$$
\mathfrak{p}(z) \prec \mathfrak{q}(z)
$$

or, equivalently,

$$
z^{p} H_{p, q, s}^{\lambda, \mu}\left(\beta_{1}+1\right) f(z) \prec \mathfrak{q}(z),
$$

which evidently completes the proof of Theorem 12 .

Our next result is an extension of Theorem 12 to the case where the behavior of $\mathfrak{q}(z)$ on $\partial \mathbb{U}$ is not known.

Corollary 13. Let $\Omega \subset \mathbb{C}$ and let the function $\mathfrak{q}$ be univalent in $\mathbb{U}$ with $\mathfrak{q}(0)=1$. Suppose also that $\phi \in \Phi_{H}\left[\Omega, \mathfrak{q}_{\rho}\right]$ for some $\rho \in(0,1)$, where $\mathfrak{q}_{\rho}(z)=\mathfrak{q}(\rho z)$. If the functions $f \in \Sigma_{p}$ and $\mathfrak{q}_{\rho}$ satisfy the following conditions:

$$
\begin{gathered}
\mathfrak{R}\left(\frac{\xi \mathfrak{q}_{\rho}^{\prime \prime}(\xi)}{\mathfrak{q}_{\rho}^{\prime}(\xi)}\right) \geqq 0, \\
\left|\frac{z^{p} H_{p, q, s}^{\lambda, \mu}\left(\beta_{1}\right) f(z)}{\mathfrak{q}_{\rho}^{\prime}(\xi)}\right| \leqq k \quad\left(z \in \mathbb{U} ; \xi \in \partial \mathbb{U} \backslash E\left(\mathfrak{q}_{\rho}\right)\right), \\
\phi\left(z^{p} H_{p, q, s}^{\lambda, \mu}\left(\beta_{1}+1\right) f(z), z^{p} H_{p, q, s}^{\lambda, \mu}\left(\beta_{1}\right) f(z),\right. \\
z^{p} H_{p, q, s}^{\lambda, \mu}\left(\beta_{1}-1\right) f(z), \\
\left.z^{p} H_{p, q, s}^{\lambda, \mu}\left(\beta_{1}-2\right) f(z) ; z\right) \in \Omega,
\end{gathered}
$$

then

$$
z^{p} H_{p, q, s}^{\lambda, \mu}\left(\beta_{1}+1\right) f(z) \prec \mathfrak{q}(z) .
$$

Proof. We note from Theorem 12 that

$$
z^{p} H_{p, q, s}^{\lambda, \mu}\left(\beta_{1}+1\right) f(z) \prec \mathfrak{q}_{\rho}(z)
$$

The result asserted by Corollary 13 is now deduced from the following subordination property:

$$
\mathfrak{q}_{\rho}(z) \prec \mathfrak{q}(z) .
$$


If $\Omega \neq \mathbb{C}$ is a simply connected domain, then $\Omega=h(\mathbb{U})$ for some conformal mapping $h(z)$ of $\mathbb{U}$ onto $\Omega$. In this case, the class $\Phi_{H}[h(\mathbb{U}), \mathfrak{q}]$ is written as $\Phi_{H}[h, \mathfrak{q}]$. The following two results are immediate consequences of Theorem 12 and Corollary 13.

Theorem 14. Let $\phi \in \Phi_{H}[h, \mathfrak{q}]$. If the functions $f \in \Sigma_{p}$ and $\mathfrak{q} \in \mathbb{Q}_{1}$ satisfy the following conditions:

$$
\begin{gathered}
\mathfrak{R}\left(\frac{\xi \mathfrak{q}^{\prime \prime}(\xi)}{\mathfrak{q}^{\prime}(\xi)}\right) \geqq 0, \quad\left|\frac{z^{p} H_{p, q, s}^{\lambda, \mu}\left(\beta_{1}\right) f(z)}{\mathfrak{q}^{\prime}(\xi)}\right| \leqq k, \\
\phi\left(z^{p} H_{p, q, s}^{\lambda, \mu}\left(\beta_{1}+1\right) f(z), z^{p} H_{p, q, s}^{\lambda, \mu}\left(\beta_{1}\right) f(z),\right. \\
z^{p} H_{p, q, s}^{\lambda, \mu}\left(\beta_{1}-1\right) f(z), \\
\left.z^{p} H_{p, q, s}^{\lambda, \mu}\left(\beta_{1}-2\right) f(z) ; z\right) \prec h(z),
\end{gathered}
$$

then

$$
z^{p} H_{p, q, s}^{\lambda, \mu}\left(\beta_{1}+1\right) f(z) \prec \mathfrak{q}(z) .
$$

Corollary 15. Let $\Omega \subset \mathbb{C}$ and let the function $\mathfrak{q}$ be univalent in $\mathbb{U}$ with $\mathfrak{q}(0)=1$. Suppose also that $\phi \in \Phi_{H}\left[h, \mathfrak{q}_{\rho}\right]$ for some $\rho \in(0,1)$, where $\mathfrak{q}_{\rho}(z)=\mathfrak{q}(\rho z)$. If the functions $f \in \Sigma_{p}$ and $\mathfrak{q}_{\rho}$ satisfy the following conditions:

$$
\begin{gathered}
\mathfrak{R}\left(\frac{\xi \mathfrak{q}_{\rho}^{\prime \prime}(\xi)}{\mathfrak{q}_{\rho}^{\prime}(\xi)}\right) \geqq 0, \\
\left|\frac{z^{p} H_{p, q, s}^{\lambda, \mu}\left(\beta_{1}\right) f(z)}{\mathfrak{q}_{\rho}^{\prime}(\xi)}\right| \leqq k \quad\left(z \in \mathbb{U} ; \xi \in \partial \mathbb{U} \backslash E\left(\mathfrak{q}_{\rho}\right)\right), \\
\phi\left(z^{p} H_{p, q, s}^{\lambda, \mu}\left(\beta_{1}+1\right) f(z), z^{p} H_{p, q, s}^{\lambda, \mu}\left(\beta_{1}\right) f(z),\right. \\
z^{p} H_{p, q, s}^{\lambda, \mu}\left(\beta_{1}-1\right) f(z), \\
\left.z^{p} H_{p, q, s}^{\lambda, \mu}\left(\beta_{1}-2\right) f(z) ; z\right)<h(z),
\end{gathered}
$$

then

$$
z^{p} H_{p, q, s}^{\lambda, \mu}\left(\beta_{1}+1\right) f(z) \prec \mathfrak{q}(z) .
$$

Our next theorem yields the best dominant of the differential subordination (70).

Theorem 16. Let the function $h$ be univalent in $\mathbb{U}$. Also let $\phi: \mathbb{C}^{4} \times \mathbb{U} \rightarrow \mathbb{C}$ and $\psi$ be given by (70). Suppose that the differential equation

$$
\psi\left(\mathfrak{q}(z), z \mathfrak{q}^{\prime}(z), z^{2} \mathfrak{q}^{\prime \prime}(z), z^{3} \mathfrak{q}^{\prime \prime \prime}(z) ; z\right)=h(z)
$$

has a solution $\mathfrak{q}(z)$ with $\mathfrak{q}(0)=1$, which satisfies condition (61). If the function $f \in \Sigma_{p}$ satisfies condition (81) and the function

$$
\begin{aligned}
& \phi\left(z^{p} H_{p, q, s}^{\lambda, \mu}\left(\beta_{1}+1\right) f(z), z^{p} H_{p, q, s}^{\lambda, \mu}\left(\beta_{1}\right) f(z),\right. \\
& \left.\quad z^{p} H_{p, q, s}^{\lambda, \mu}\left(\beta_{1}-1\right) f(z), z^{p} H_{p, q, s}^{\lambda, \mu}\left(\beta_{1}-2\right) f(z) ; z\right)
\end{aligned}
$$

is analytic in $\mathbb{U}$, then

$$
z^{p} H_{p, q, s}^{\lambda, \mu}\left(\beta_{1}+1\right) f(z) \prec \mathfrak{q}(z)
$$

and $\mathfrak{q}(z)$ is the best dominant.

Proof. By applying Theorem 12, we deduce that $\mathfrak{q}$ is a dominant of (81). Since $\mathfrak{q}$ satisfies (85), it is also a solution of (81). Therefore, $\mathfrak{q}$ will be dominated by all dominants. Hence $\mathfrak{q}$ is the best dominant.

In view of Definition 11, in the particular case when $\mathfrak{q}(z)=$ $1+M z(M>0)$, the class $\Phi_{H}[\Omega, \mathfrak{q}]$ of admissible functions, denoted simply by $\Phi_{H}[\Omega, M]$, is described below.

Definition 17. Let $\Omega$ be a set in $\mathbb{C}, \beta_{1} \in \mathbb{C} \backslash\{0,-1,-2, \ldots\}$, and $M>0$. The class $\Phi_{H}[\Omega, M]$ of admissible functions consists of those functions $\phi: \mathbb{C}^{4} \times \mathbb{U} \rightarrow \mathbb{C}$ such that

$$
\begin{aligned}
& \phi\left(1+M e^{i \theta}, 1+\frac{k+\beta_{1}}{\beta_{1}} M e^{i \theta},\right. \\
& 1+\frac{L+\left(2 k+\beta_{1}\right)\left(\beta_{1}+1\right) M e^{i \theta}}{\beta_{1}\left(\beta_{1}+1\right)}, \\
& \left.1+\frac{N+3\left(\beta_{1}+2\right) L+\left(3 k+\beta_{1}\right)\left(\beta_{1}+1\right)\left(\beta_{1}+2\right) M e^{i \theta}}{\beta_{1}\left(\beta_{1}+1\right)\left(\beta_{1}+2\right)} ; z\right)
\end{aligned}
$$

$\notin \Omega$

whenever $z \in \mathbb{U}, \mathfrak{R}\left(L e^{-i \theta}\right) \geqq(k-1) k M$ and $\mathfrak{R}\left(N e^{-i \theta}\right) \geqq 0$ for all $\theta \in \mathbb{R}$ and $k \in \mathbb{N} \backslash\{1\}$.

Corollary 18. Let $\phi \in \Phi_{H}[\Omega, M]$. If the function $f \in \Sigma_{p}$ satisfies the following conditions:

$$
\begin{gathered}
\left|z^{p} H_{p, q, s}^{\lambda, \mu}\left(\beta_{1}\right) f(z)\right| \leqq k M \quad(k \in \mathbb{N} \backslash\{1\} ; M>0), \\
\phi\left(z^{p} H_{p, q, s}^{\lambda, \mu}\left(\beta_{1}+1\right) f(z), z^{p} H_{p, q, s}^{\lambda, \mu}\left(\beta_{1}\right) f(z),\right. \\
z^{p} H_{p, q, s}^{\lambda, \mu}\left(\beta_{1}-1\right) f(z), \\
\left.z^{p} H_{p, q, s}^{\lambda, \mu}\left(\beta_{1}-2\right) f(z) ; z\right) \in \Omega,
\end{gathered}
$$

then

$$
\left|z^{p} H_{p, q, s}^{\lambda, \mu}\left(\beta_{1}+1\right) f(z)-1\right|<M \quad(M>0) .
$$

In the special case when

$$
\Omega=\mathfrak{q}(\mathbb{U})=\{\omega:|\omega-1|<M(M>0)\},
$$

the class $\Phi_{H}[\Omega, M]$ is denoted, for brevity, by $\Phi_{H}[M]$. Corollary 18 can now be rewritten in the following form. 
Corollary 19. Let $\phi \in \Phi_{H}[M]$. If the function $f \in \Sigma_{p}$ satisfies the following conditions:

$$
\begin{gathered}
\left|z^{p} H_{p, q, s}^{\lambda, \mu}\left(\beta_{1}\right) f(z)\right| \leqq k M \quad(k \in \mathbb{N} \backslash\{1\} ; M>0), \\
\mid \phi\left(z^{p} H_{p, q, s}^{\lambda, \mu}\left(\beta_{1}+1\right) f(z), z^{p} H_{p, q, s}^{\lambda, \mu}\left(\beta_{1}\right) f(z),\right. \\
z^{p} H_{p, q, s}^{\lambda, \mu}\left(\beta_{1}-1\right) f(z), \\
\left.z^{p} H_{p, q, s}^{\lambda, \mu}\left(\beta_{1}-2\right) f(z) ; z\right)-1 \mid<M,
\end{gathered}
$$

then

$$
\left|z^{p} H_{p, q, s}^{\lambda, \mu}\left(\beta_{1}+1\right) f(z)-1\right|<M \quad(M>0) .
$$

Corollary 20. Let $\beta_{1} \in \mathbb{C} \backslash\{0,-1,-2, \ldots\}$ with $\mathfrak{R}\left(\beta_{1}\right) \geqq-1 / 2$ and $M>0$. If the function $f \in \Sigma_{p}$ satisfies the following conditions:

$$
\begin{gathered}
\left|z^{p} H_{p, q, s}^{\lambda, \mu}\left(\beta_{1}\right) f(z)\right| \leqq k M \quad(k \in \mathbb{N} \backslash\{1\}), \\
\left|z^{p} H_{p, q, s}^{\lambda, \mu}\left(\beta_{1}\right) f(z)-1\right|<M,
\end{gathered}
$$

then

$$
\left|z^{p} H_{p, q, s}^{\lambda, \mu}\left(\beta_{1}+1\right) f(z)-1\right|<M .
$$

Proof. Corollary 20 follows from Corollary 19 by setting

$$
\phi(a, b, c, d ; z)=b=1+\frac{k+\beta_{1}}{\beta_{1}} M e^{i \theta} .
$$

Corollary 21. Let $\beta_{1} \in \mathbb{C} \backslash\{0,-1,-2, \ldots\}, k \in \mathbb{N} \backslash\{1\}$, and $M>0$. If the function $f \in \Sigma_{p}$ satisfies the following conditions:

$$
\begin{gathered}
\left|z^{p} H_{p, q, s}^{\lambda, \mu}\left(\beta_{1}\right) f(z)\right| \leqq k M, \\
\left|z^{p} H_{p, q, s}^{\lambda, \mu}\left(\beta_{1}-2\right) f(z)-z^{p} H_{p, q, s}^{\lambda, \mu}\left(\beta_{1}-1\right) f(z)\right|<\frac{M}{\left|\beta_{1}\right|},
\end{gathered}
$$

then

$$
\left|z^{p} H_{p, q, s}^{\lambda, \mu}\left(\beta_{1}+1\right) f(z)-1\right|<M
$$

Proof. Let

$$
\phi(a, b, c, d ; z)=d-c, \quad \Omega=h(\mathbb{U}),
$$

where

$$
h(z)=\frac{M z}{\left|\beta_{1}\right|} \quad(M>0) .
$$

In order to use Corollary 18, we need to show that $\phi \epsilon$ $\Phi_{H}[\Omega, M]$; that is, the admissibility condition (88) is satisfied. This follows easily, since

$$
\begin{aligned}
\mid \phi & \left(1+M e^{i \theta}, 1+\frac{k+\beta_{1}}{\beta_{1}} M e^{i \theta},\right. \\
& 1+\frac{L+\left(2 k+\beta_{1}\right)\left(\beta_{1}+1\right) M e^{i \theta}}{\beta_{1}\left(\beta_{1}+1\right)}, \\
& \left.1+\frac{N+3\left(\beta_{1}+2\right) L+\left(3 k+\beta_{1}\right)\left(\beta_{1}+1\right)\left(\beta_{1}+2\right) M e^{i \theta}}{\beta_{1}\left(\beta_{1}+1\right)\left(\beta_{1}+2\right)} ; z\right) \mid \\
= & \mid \frac{N+3\left(\beta_{1}+2\right) L+\left(3 k+\beta_{1}\right)\left(\beta_{1}+1\right)\left(\beta_{1}+2\right) M e^{i \theta}}{\beta_{1}\left(\beta_{1}+1\right)\left(\beta_{1}+2\right)} \\
= & \left|\frac{N e^{-i \theta}+2\left(\beta_{1}+2\right) L e^{-i \theta}+k\left(\beta_{1}+1\right)\left(\beta_{1}+2\right) M}{\beta_{1}\left(\beta_{1}+1\right)\left(\beta_{1}+2\right) e^{-i \theta}}\right|
\end{aligned}
$$$$
\geqq \frac{\Re\left(N e^{-i \theta}\right)+2\left|\beta_{1}+2\right| \Re\left(L e^{-i \theta}\right)+k\left|\beta_{1}+1\right|\left|\beta_{1}+2\right| M}{\left|\beta_{1}\left(\beta_{1}+1\right)\left(\beta_{1}+2\right)\right|}
$$$$
\geqq \frac{k\left(2 k-2+\left|\beta_{1}+1\right|\right) M}{\left|\beta_{1}\right|\left|\beta_{1}+1\right|}
$$$$
\geqq \frac{M}{\left|\beta_{1}\right|}
$$

whenever $z \in \mathbb{U}, \mathfrak{R}\left(L e^{-i \theta}\right) \geqq(k-1) k M$, and $\mathfrak{R}\left(N e^{-i \theta}\right) \geqq 0$ for all $\theta \in \mathbb{R}$ and $k \in \mathbb{N} \backslash\{1\}$. The required result now follows from Corollary 18.

\section{Third-Order Differential Superordination of the Operator $H_{p, q, s}^{\lambda, \mu}\left(\beta_{1}\right)$}

In this section, we obtain the third-order differential superordination results for meromorphically multivalent functions associated with the operator $H_{p, q, s}^{\lambda, \mu}\left(\beta_{1}\right)$ defined by (16). Because of this, the class of admissible functions is given in the following definition.

Definition 22. Let $\Omega$ be a set in $\mathbb{C}$ and $\mathfrak{q} \in \mathscr{H}$ with $\mathfrak{q}^{\prime}(z) \neq 0$. The class of admissible functions $\Phi_{H}^{\prime}[\Omega, \mathfrak{q}]$ consists of those functions $\phi: \mathbb{C}^{4} \times \overline{\mathbb{U}} \rightarrow \mathbb{C}$ that satisfy the following admissibility condition:

$$
\phi(a, b, c, d ; \xi) \in \Omega
$$


whenever

$$
\begin{gathered}
a=\mathfrak{q}(z), \quad b=\frac{z \mathfrak{q}^{\prime}(z)+m \beta_{1} \mathfrak{q}(z)}{m \beta_{1}}, \\
\Re\left(\frac{\left(\beta_{1}+1\right)(c-a)}{b-a}-\left(2 \beta_{1}+1\right)\right) \\
\leqq \frac{1}{m} \mathfrak{R}\left\{\frac{z \mathfrak{q}^{\prime \prime}(z)}{\mathfrak{q}^{\prime}(z)}+1\right\}, \\
\Re\left(\frac{\left(\beta_{1}+1\right)\left(\beta_{1}+2\right)(d-3 c+3 b-a)}{b-a}\right) \\
\leqq \frac{1}{m^{2}} \mathfrak{R}\left\{\frac{z^{2} \mathfrak{q}^{\prime \prime \prime}(z)}{\mathfrak{q}^{\prime}(z)}\right\},
\end{gathered}
$$

where $z \in \mathbb{U}, \beta_{1} \in \mathbb{C} \backslash\{0,-1,-2, \ldots\}, \xi \in \partial \mathbb{U}$, and $m \in \mathbb{N} \backslash\{1\}$.

Theorem 23. Let $\phi \in \Phi_{H}^{\prime}[\Omega, \mathfrak{q}]$. If the functions $f \in \Sigma_{p}$ and $z^{p} H_{p, q, s}^{\lambda, \mu}\left(\beta_{1}+1\right) f(z) \in Q_{1}$ satisfy the following conditions:

$$
\begin{aligned}
& \Re\left(\frac{z \mathfrak{q}^{\prime \prime}(z)}{\mathfrak{q}^{\prime}(z)}\right) \geqq 0, \quad\left|\frac{z^{p} H_{p, q, s}^{\lambda, \mu}\left(\beta_{1}\right) f(z)}{\mathfrak{q}^{\prime}(z)}\right| \leqq m, \quad(10) \\
& \phi\left(z^{p} H_{p, q, s}^{\lambda, \mu}\left(\beta_{1}+1\right) f(z), z^{p} H_{p, q, s}^{\lambda, \mu}\left(\beta_{1}\right) f(z),\right. \\
& \left.z^{p} H_{p, q, s}^{\lambda, \mu}\left(\beta_{1}-1\right) f(z), z^{p} H_{p, q, s}^{\lambda, \mu}\left(\beta_{1}-2\right) f(z) ; z\right)
\end{aligned}
$$

is univalent

$$
\begin{gathered}
\Omega \subset\left\{\phi \left(z^{p} H_{p, q, s}^{\lambda, \mu}\left(\beta_{1}+1\right) f(z),\right.\right. \\
z^{p} H_{p, q, s}^{\lambda, \mu}\left(\beta_{1}\right) f(z), z^{p} H_{p, q, s}^{\lambda, \mu}\left(\beta_{1}-1\right) f(z), \\
\left.\left.z^{p} H_{p, q, s}^{\lambda, \mu}\left(\beta_{1}-2\right) f(z) ; z\right): z \in \mathbb{U}\right\}
\end{gathered}
$$

implies that

$$
\mathfrak{q}(z) \prec z^{p} H_{p, q, s}^{\lambda, \mu}\left(\beta_{1}+1\right) f(z) .
$$

Proof. Let the function $\mathfrak{p}(z)$ be defined by (64) and $\psi$ by (70). Since $\phi \in \Phi_{H}^{\prime}[\Omega, \mathfrak{q}],(71)$ and (106) yield

$$
\Omega \subset\left\{\psi\left(\mathfrak{p}(z), z \mathfrak{p}^{\prime}(z), z^{2} \mathfrak{p}^{\prime \prime}(z), z^{3} \mathfrak{p}^{\prime \prime \prime}(z) ; z\right): z \in \mathbb{U}\right\} .
$$

We see from (68) and (69) that the admissible condition for $\phi \in \Phi_{H}^{\prime}[\Omega, \mathfrak{q}]$ in Definition 22 is equivalent to the admissible condition for $\psi$ as given in Definition 7 with $n=2$. Hence $\psi \in \Psi_{2}^{\prime}[\Omega, \mathfrak{q}]$, and by using (104) and Theorem 8 , we have

$$
\mathfrak{q}(z) \prec \mathfrak{p}(z)
$$

or, equivalently,

$$
\mathfrak{q}(z) \prec z^{p} H_{p, q, s}^{\lambda, \mu}\left(\beta_{1}+1\right) f(z),
$$

which evidently completes the proof of Theorem 23 .
If $\Omega \neq \mathbb{C}$ is a simply connected domain and $\Omega=h(\mathbb{U})$ for some conformal mapping $h(z)$ of $\mathbb{U}$ onto $\Omega$, then the class $\Phi_{H}^{\prime}[h(\mathbb{U}), \mathfrak{q}]$ is written simply as $\Phi_{H}^{\prime}[h, \mathfrak{q}]$. With proceedings similar as in the preceding section, the following result is an immediate consequence of Theorem 23.

Theorem 24. Let $\phi \in \Phi_{H}^{\prime}[h, \mathfrak{q}]$. Also let the function $h$ be analytic in $\mathbb{U}$. If the functions $f \in \Sigma_{p}$ and $z^{p} H_{p, q, s}^{\lambda, \mu}\left(\beta_{1}+\right.$ 1) $f(z) \in Q_{1}$ satisfy condition (104) and

$$
\begin{aligned}
\phi\left(z^{p} H_{p, q, s}^{\lambda, \mu}\left(\beta_{1}+1\right) f(z), z^{p} H_{p, q, s}^{\lambda, \mu}\left(\beta_{1}\right) f(z),\right. \\
\left.z^{p} H_{p, q, s}^{\lambda, \mu}\left(\beta_{1}-1\right) f(z), z^{p} H_{p, q, s}^{\lambda, \mu}\left(\beta_{1}-2\right) f(z) ; z\right)
\end{aligned}
$$

is univalent in $\mathbb{U}$, then

$$
\begin{gathered}
h(z) \prec \phi\left(z^{p} H_{p, q, s}^{\lambda, \mu}\left(\beta_{1}+1\right) f(z), z^{p} H_{p, q, s}^{\lambda, \mu}\left(\beta_{1}\right) f(z),\right. \\
z^{p} H_{p, q, s}^{\lambda, \mu}\left(\beta_{1}-1\right) f(z), \\
\left.z^{p} H_{p, q, s}^{\lambda, \mu}\left(\beta_{1}-2\right) f(z) ; z\right)
\end{gathered}
$$

implies that

$$
\mathfrak{q}(z) \prec z^{p} H_{p, q, s}^{\lambda, \mu}\left(\beta_{1}+1\right) f(z) .
$$

Theorems 23 and 24 can only be used to obtain subordinations involving the third-order differential superordination of the forms (106) or (112). The following theorem proves the existence of the best subordinant of (112) for a suitable chosen $\phi$.

Theorem 25. Let the function $h$ be analytic in $\mathbb{U}$, and let $\phi: \mathbb{C}^{4} \times \overline{\mathbb{U}} \rightarrow \mathbb{C}$ and $\psi$ be given by (70). Suppose that the differential equation

$$
\psi\left(\mathfrak{q}(z), z \mathfrak{q}^{\prime}(z), z^{2} \mathfrak{q}^{\prime \prime}(z), z^{3} \mathfrak{q}^{\prime \prime \prime}(z) ; z\right)=h(z)
$$

has a solution $\mathfrak{q}(z) \in Q_{1}$. If the functions $f \in \Sigma_{p}$ and $z^{p} H_{p, q, s}^{\lambda, \mu}\left(\beta_{1}+1\right) f(z) \in Q_{1}$ satisfy condition (104) and

$$
\begin{aligned}
& \phi\left(z^{p} H_{p, q, s}^{\lambda, \mu}\left(\beta_{1}+1\right) f(z), z^{p} H_{p, q, s}^{\lambda, \mu}\left(\beta_{1}\right) f(z),\right. \\
& \left.z^{p} H_{p, q, s}^{\lambda, \mu}\left(\beta_{1}-1\right) f(z), z^{p} H_{p, q, s}^{\lambda, \mu}\left(\beta_{1}-2\right) f(z) ; z\right),
\end{aligned}
$$

is univalent in $\mathbb{U}$, then

$$
\begin{gathered}
h(z) \prec \phi\left(z^{p} H_{p, q, s}^{\lambda, \mu}\left(\beta_{1}+1\right) f(z), z^{p} H_{p, q, s}^{\lambda, \mu}\left(\beta_{1}\right) f(z),\right. \\
z^{p} H_{p, q, s}^{\lambda, \mu}\left(\beta_{1}-1\right) f(z), \\
\left.z^{p} H_{p, q, s}^{\lambda, \mu}\left(\beta_{1}-2\right) f(z) ; z\right)
\end{gathered}
$$

implies that

$$
\mathfrak{q}(z) \prec z^{p} H_{p, q, s}^{\lambda, \mu}\left(\beta_{1}+1\right) f(z)
$$

and $\mathfrak{q}$ is the best subordinant. 
Proof. The proof of Theorem 25 is similar to that of Theorem 16 and it is being omitted here.

By combining Theorems 14 and 24, we obtain the following sandwich-type result.

Corollary 26. Let the functions $h_{1}$ and $\mathfrak{q}_{1}$ be analytic functions in $\mathbb{U}$. Also let the function $h_{2}$ be univalent in $\mathbb{U}, \mathfrak{q}_{2} \in \mathbb{Q}_{1}$ with $\mathfrak{q}_{1}(0)=\mathfrak{q}_{2}(0)=1$ and $\phi \in \Phi_{H}\left[h_{2}, \mathfrak{q}_{2}\right] \cap \Phi_{H}^{\prime}\left[h_{1}, \mathfrak{q}_{1}\right]$. If the function $f \in \Sigma_{p}$, $z^{p} H_{p, q, s}^{\lambda, \mu}\left(\beta_{1}+1\right) f(z) \in \mathbb{Q}_{1} \cap \mathscr{H}$ and

$$
\begin{aligned}
\phi\left(z^{p} H_{p, q, s}^{\lambda, \mu}\left(\beta_{1}+1\right) f(z), z^{p} H_{p, q, s}^{\lambda, \mu}\left(\beta_{1}\right) f(z),\right. \\
\left.z^{p} H_{p, q, s}^{\lambda, \mu}\left(\beta_{1}-1\right) f(z), z^{p} H_{p, q, s}^{\lambda, \mu}\left(\beta_{1}-2\right) f(z) ; z\right)
\end{aligned}
$$

is univalent in $\mathbb{U}$, and the conditions (61) and (104) are satisfied, then

$$
\begin{gathered}
h_{1}(z) \prec \phi\left(z^{p} H_{p, q, s}^{\lambda, \mu}\left(\beta_{1}+1\right) f(z), z^{p} H_{p, q, s}^{\lambda, \mu}\left(\beta_{1}\right) f(z),\right. \\
z^{p} H_{p, q, s}^{\lambda, \mu}\left(\beta_{1}-1\right) f(z), \\
\left.z^{p} H_{p, q, s}^{\lambda, \mu}\left(\beta_{1}-2\right) f(z) ; z\right) \prec h_{2}(z)
\end{gathered}
$$

implies that

$$
\mathfrak{q}_{1}(z) \prec z^{p} H_{p, q, s}^{\lambda, \mu}\left(\beta_{1}+1\right) f(z) \prec \mathfrak{q}_{2}(z) .
$$

Remark 27. By setting $\lambda=\mu=0$ in all results of this paper, we can obtain the corresponding results for the well-known Liu-Srivastava operator $H_{p, q, s}\left(\beta_{1}\right)$.

\section{Concluding Remarks and Observations}

In our present investigation, we have derived several thirdorder differential subordination and superordination results for meromorphically multivalent functions in the punctured unit disk involving the operator $H_{p, q, s}^{\lambda, \mu}\left(\beta_{1}\right)$ defined by (16) with respect to the parameter $\beta_{1} \in \mathbb{C} \backslash\{0,-1,-2, \ldots\}$, which is associated with the Liu-Srivastava operator $H_{p, q, s}\left(\beta_{1}\right)$. Our results have been obtained by considering suitable classes of admissible functions. Furthermore, if we use relation (19), we can obtain the corresponding third-order differential subordination and superordination results for the operator $H_{p, q, s}^{\lambda, \mu}\left(\alpha_{1}\right)$ with respect to the parameter $\alpha_{1} \in \mathbb{C}$ and here we choose to omit the details involved.

\section{Conflict of Interests}

The authors declare that there is no conflict of interests regarding the publication of this paper.

\section{Acknowledgments}

The research was partly supported by the Natural Science Foundation of China under Grant 11271045, the Higher
School Doctoral Foundation of China under Grant 20100003110004, the Natural Science Foundation of Inner Mongolia of China under Grant 2010MS0117, and the Higher School Foundation of Inner Mongolia of China under Grant NJZY13298. The authors would like to thank Professors Om P. Ahuja and V. Ravichandran for their valuable suggestions and the referees for their careful reading and helpful comments to improve their paper.

\section{References}

[1] S. S. Miller and P. T. Mocanu, Differential Subordinations: Theory and Applications, vol. 225 of Series on Monographs and Textbooks in Pure and Applied Mathematics, No. 225, Marcel Dekker Incorporated, New York, NY, USA, 2000.

[2] S. Owa and H. M. Srivastava, "Univalent and starlike generalized hypergeometric functions," Canadian Journal of Mathematics, vol. 39, no. 5, pp. 1057-1077, 1987.

[3] H. M. Srivastava and S. Owa, "Some characterization and distortion theorems involving fractional calculus, generalized hypergeometric functions, Hadamard products, linear operators, and certain subclasses of analytic functions," Nagoya Mathematical Journal, vol. 106, pp. 1-28, 1987.

[4] H. Tang, M. K. Aouf, and G. T. Deng, "Majorization problems for certainsubclasses of meromorphic multivalent functions associated with the Liu-Srivastava operator," Filomat. In press.

[5] J.-L. Liu and H. M. Srivastava, "Classes of meromorphically multivalent functions associated with the generalized hypergeometric function," Mathematical and Computer Modelling, vol. 39, no. 1, pp. 21-34, 2004.

[6] J.-L. Liu and H. M. Srivastava, "Subclasses of meromorphically multivalent functions associated with a certain linear operator," Mathematical and Computer Modelling, vol. 39, no. 1, pp. 35-44, 2004.

[7] N. E. Cho, O. S. Kwon, and H. M. Srivastava, "Strong differential subordination and superordination for multivalently meromorphic functions involving the Liu-Srivastava operator," Integral Transforms and Special Functions, vol. 21, no. 7-8, pp. 589-601, 2010.

[8] J. Dziok and H. M. Srivastava, "Classes of analytic functions associated with the generalized hypergeometric function," Applied Mathematics and Computation, vol. 103, no. 1, pp. 1-13, 1999.

[9] J. Dziok and H. M. Srivastava, "Some subclasses of analytic functions with fixed argument of coefficients associated with the generalized hypergeometric function," Advanced Studies in Contemporary Mathematics, vol. 5, no. 2, pp. 115-125, 2002.

[10] J. Dziok and H. M. Srivastava, "Certain subclasses of analytic functions associated with the generalized hypergeometric function," Integral Transforms and Special Functions, vol. 14, no. 1, pp. $7-18,2003$.

[11] J.-L. Liu and H. M. Srivastava, "Certain properties of the DziokSrivastava operator," Applied Mathematics and Computation, vol. 159, no. 2, pp. 485-493, 2004.

[12] J. Patel, A. K. Mishra, and H. M. Srivastava, "Classes of multivalent analytic functions involving the Dziok-Srivastava operator," Computers \& Mathematics with Applications, vol. 54, no. 5, pp. 599-616, 2007.

[13] J.-L. Liu, "A linear operator and its applications on meromorphic p-valent functions," Bulletin of the Institute of Mathematics. Academia Sinica, vol. 31, no. 1, pp. 23-32, 2003. 
[14] J.-L. Liu and H. M. Srivastava, "A linear operator and associated families of meromorphically multivalent functions," Journal of Mathematical Analysis and Applications, vol. 259, no. 2, pp. 566581, 2001.

[15] D. Yang, "On a class of meromorphic starlike multivalent functions," Bulletin of the Institute of Mathematics. Academia Sinica, vol. 24, no. 2, pp. 151-157, 1996.

[16] B. A. Uralegaddi and C. Somanatha, "New criteria for meromorphic starlike univalent functions," Bulletin of the Australian Mathematical Society, vol. 43, no. 1, pp. 137-140, 1991.

[17] J. A. Antonino and S. S. Miller, "Third-order differential inequalities and subordinations in the complex plane," Complex Variables and Elliptic Equations, vol. 56, no. 5, pp. 439-454, 2011.

[18] S. S. Miller and P. T. Mocanu, "Subordinants of differential superordinations," Complex Variables. Theory and Application, vol. 48, no. 10, pp. 815-826, 2003.

[19] R. M. Ali, V. Ravichandran, and N. Seenivasagan, "Subordination and superordination on Schwarzian derivatives," Journal of Inequalities and Applications, vol. 2008, Article ID 712328, 18 pages, 2008.

[20] R. M. Ali, V. Ravichandran, and N. Seenivasagan, "On subordination and superordination of the multiplier transformation for meromorphic functions," Bulletin of the Malaysian Mathematical Sciences Society. Second Series, vol. 33, no. 2, pp. 311-324, 2010.

[21] N. E. Cho, O. S. Kwon, S. Owa, and H. M. Srivastava, "A class of integral operators preserving subordination and superordination for meromorphic functions," Applied Mathematics and Computation, vol. 193, no. 2, pp. 463-474, 2007.

[22] N. E. Cho and H. M. Srivastava, "A class of nonlinear integral operators preserving subordination and superordination," Integral Transforms and Special Functions, vol. 18, no. 1-2, pp. 95-107, 2007.

[23] T. N. Shanmugam, S. Sivasubramanian, and H. Srivastava, "Differential sandwich theorems for certain subclasses of analytic functions involving multiplier transformations," Integral Transforms and Special Functions, vol. 17, no. 12, pp. 889-899, 2006. 


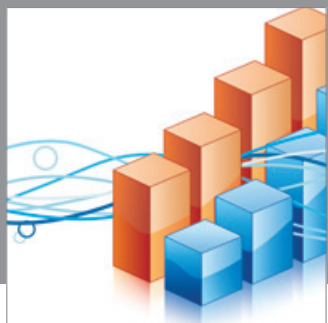

Advances in

Operations Research

mansans

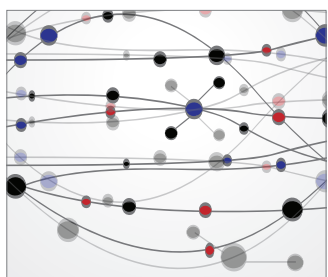

The Scientific World Journal
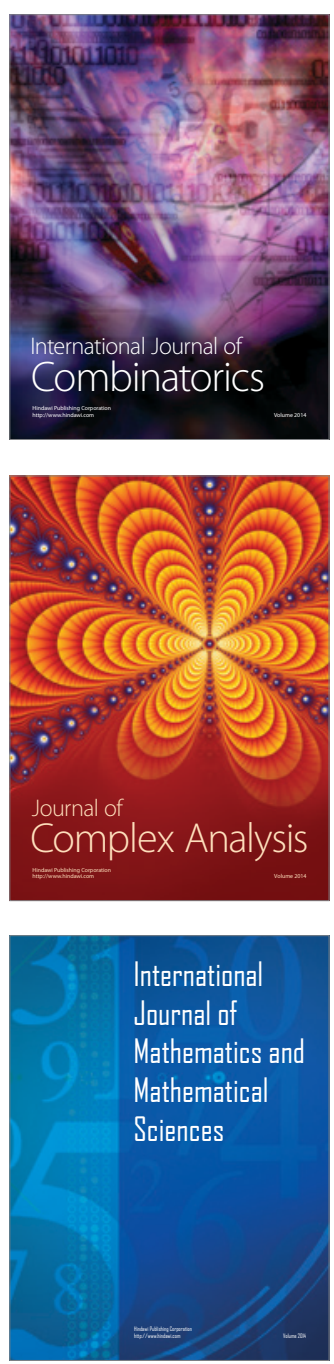
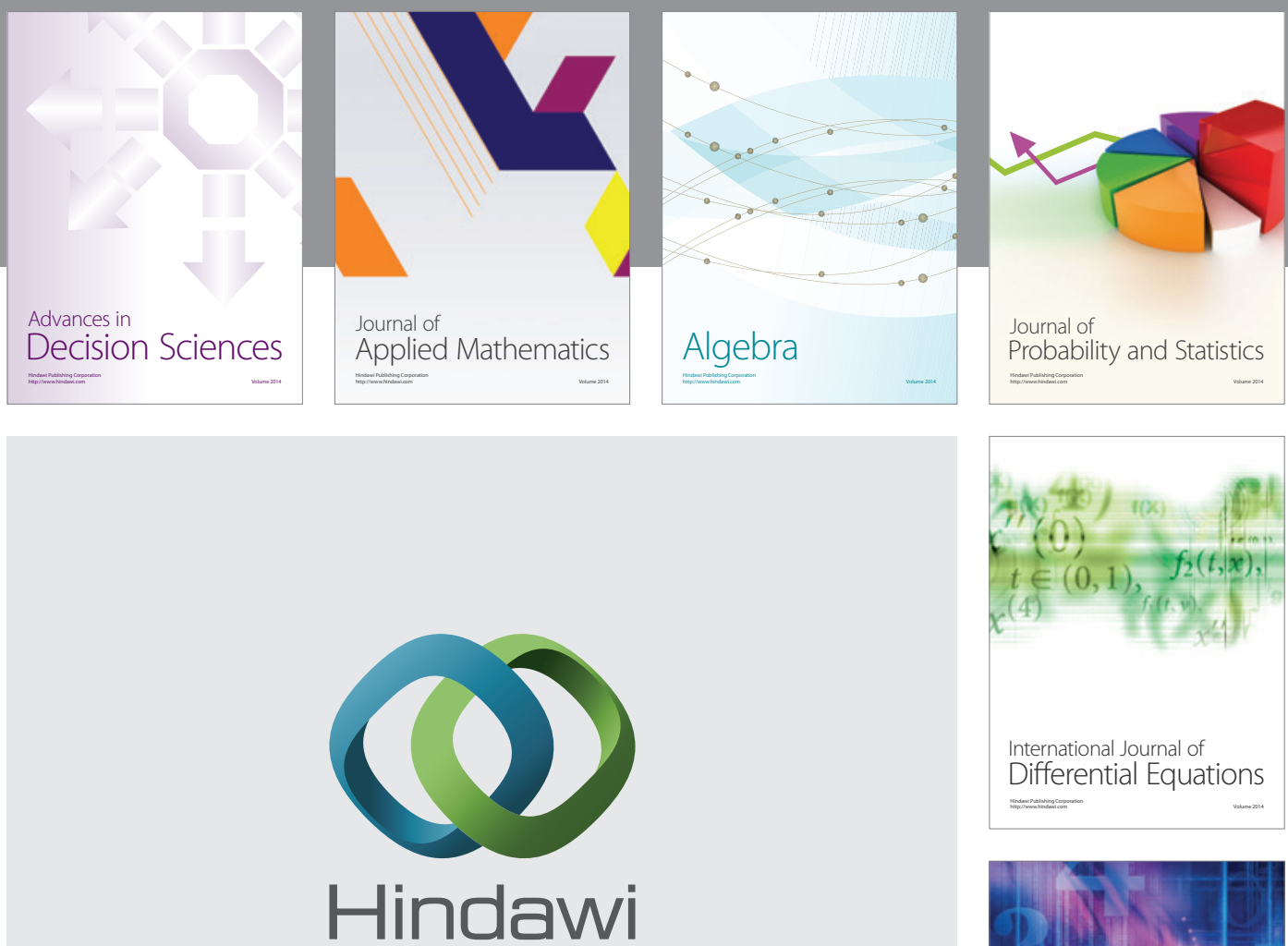

Submit your manuscripts at http://www.hindawi.com
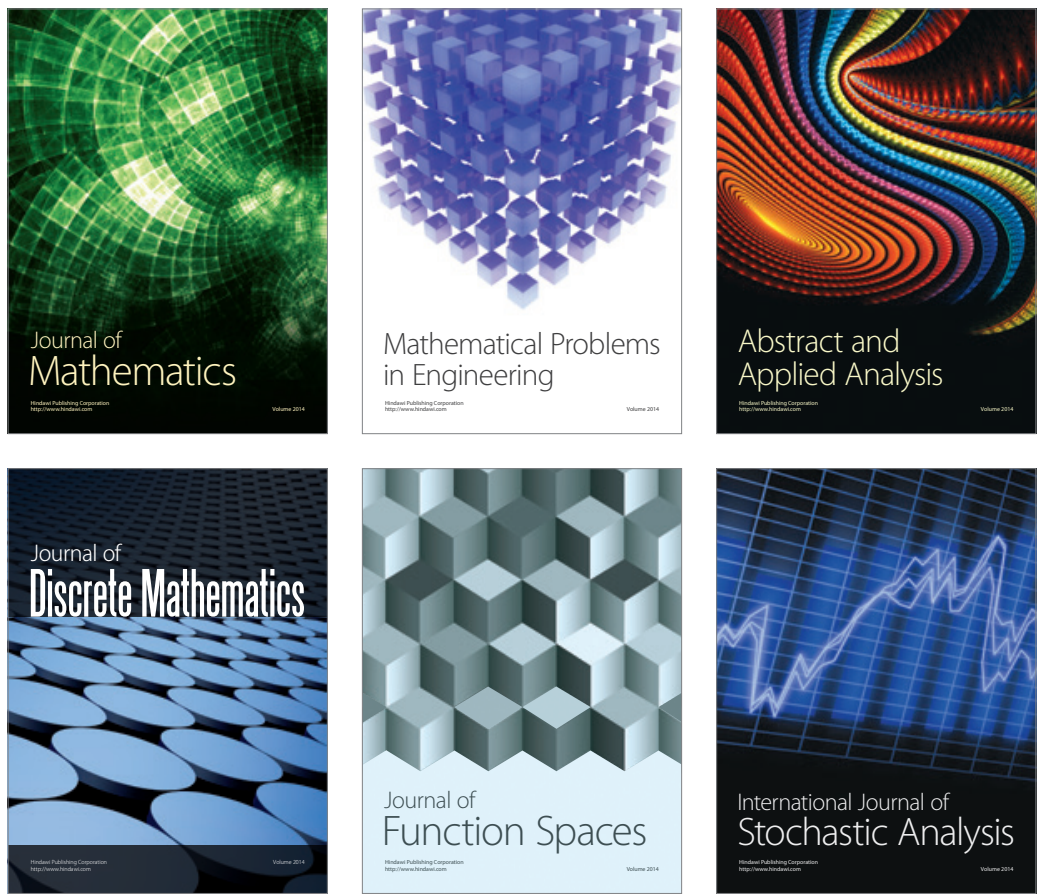

Journal of

Function Spaces

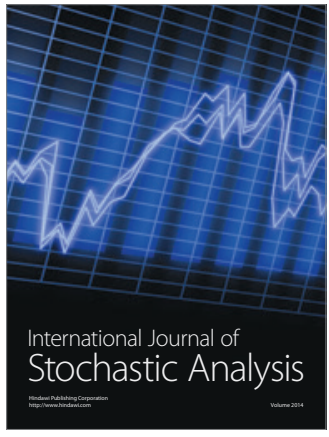

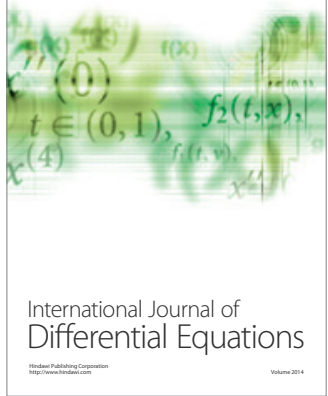
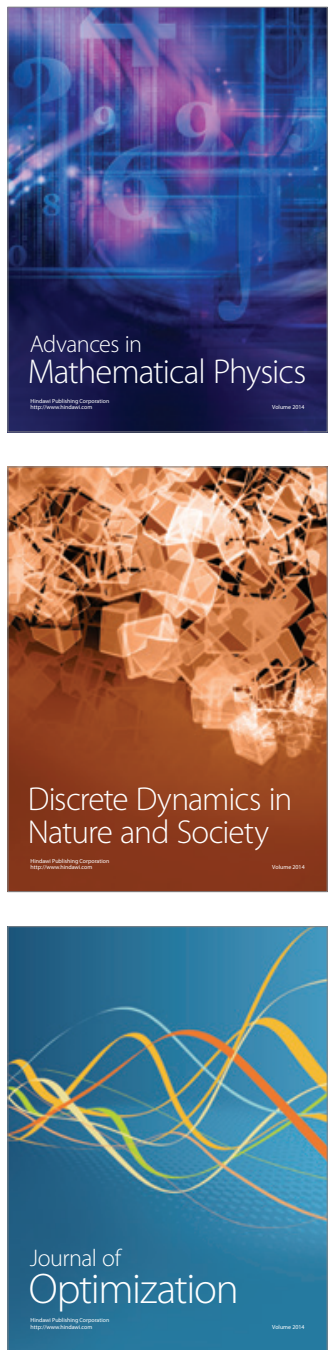